\title{
Statistics of turbulence parameters at Maunakea using the multiple wavefront sensor data of RAVEN
}

\author{
Yoshito H. Ono, ${ }^{1,2 \star}$ Carlos M. Correia, ${ }^{1}$ Dave R. Andersen, ${ }^{3}$ Olivier Lardière, ${ }^{3}$ \\ Shin Oya, ${ }^{4}$ Masayuki Akiyama, ${ }^{2}$ Kate Jackson ${ }^{6}$ and Colin Bradley ${ }^{5}$ \\ ${ }^{1}$ Aix Marseille Univ., CNRS, LAM, Laboratoire d'Astrophysique de Marseille, Marseille, France \\ ${ }^{2}$ Astronomical Institute, Tohoku University, 6-3 Aramaki, Aoba-ku, Sendai 980-8578, Japan \\ ${ }^{3}$ NRC-Herzberg, 5071 West Saanich Rd., Victoria, British Columbia, Canada \\ ${ }^{4}$ TMT-J Project Office, NAOJ, 2-21-1 Osawa, Mitaka, Tokyo 181-8588, Japan \\ ${ }^{5}$ Adaptive Optics Laboratory, University of Victoria, 3800 Finnerty Rd., Victoria V8P 5C2, British Columbia, Canada \\ ${ }^{6}$ Division of Engineering and Applied Science, California Institute of Technology, 1200 E. California Boulevard MC 155-44, Pasadena, CA 91125, USA
}

Accepted 2016 November 24. Received 2016 November 24; in original form 2016 October 17

\begin{abstract}
Prior statistical knowledge of atmospheric turbulence is essential for designing, optimizing and evaluating tomographic adaptive optics systems. We present the statistics of the vertical profiles of $C_{N}^{2}$ and the outer scale at Maunakea estimated using a SLOpe Detection And Ranging (SLODAR) method from on-sky telemetry taken by a multi-object adaptive optics (MOAO) demonstrator, called RAVEN, on the Subaru telescope. In our SLODAR method, the profiles are estimated by fitting the theoretical autocorrelations and cross-correlations of measurements from multiple Shack-Haltmann wavefront sensors to the observed correlations via the non-linear Levenberg-Marquardt Algorithm (LMA). The analytical derivatives of the spatial phase structure function with respect to its parameters for the LMA are also developed. From a total of 12 nights in the summer season, a large ground $C_{N}^{2}$ fraction of 54.3 per cent is found, with median estimated seeing of $0.460 \mathrm{arcsec}$. This median seeing value is below the results for Maunakea from the literature $(0.6-0.7 \mathrm{arcsec})$. The average $C_{N}^{2}$ profile is in good agreement with results from the literature, except for the ground layer. The median value of the outer scale is $25.5 \mathrm{~m}$ and the outer scale is larger at higher altitudes; these trends of the outer scale are consistent with findings in the literature.
\end{abstract}

Key words: atmospheric effects - instrumentation: adaptive optics - site testing.

\section{INTRODUCTION}

Prior statistical knowledge of atmospheric turbulence, such as layer altitude and stratified strength, outer scale and wind velocity (speed and direction), is essential for designing and optimizing adaptive optics (AO) systems in general, and in particular tomographic AO systems that provide corrections over fields larger than the isoplanatic angle.

Recently, wide field adaptive optics (WFAO) systems have been developed for current 8-m class telescopes (Ströbele et al. 2012; Neichel et al. 2014; Lardière et al. 2014; Vidal et al. 2014). These are being designed for future Extreme Large Telescopes (ELTs; Herriot et al. 2014; Thatte et al. 2014), which have primary mirror diameters in the range $20-40 \mathrm{~m}$. Such WFAO systems require the vertical profile of the turbulence strength $C_{N}^{2}(h)$ to tomographically reconstruct the three-dimensional structure of the phase distortion caused by the atmospheric turbulence above the telescope.

Although parameters such as the coherence length $r_{0}$, which is related to the seeing $s=0.98 \lambda / r_{0}$ (Roddier 1981), and layer heights are relatively well constrained, the estimation of the outerscale $\mathcal{L}_{0}$ with typical values of $\sim 20-30 \mathrm{~m}$ at good observation sites has become an important research topic (Ziad et al. 2004; Maire et al. 2007). As we move towards larger apertures, the impact of $\mathcal{L}_{0}$ on the estimation of seeing and $C_{N}^{2}$ becomes more important; moreover, the vertical profile of $\mathcal{L}_{0}$ makes tilt angular decorrelation very different from constant $\mathcal{L}_{0}$ profiles, thus affecting the estimation of tilt anisoplanatism and constraining system designs. The estimation of wind speed and direction $v$ can be used in advanced temporal control of AO systems (Correia et al. 2014; Ono et al. 2016). In addition, knowledge of the atmospheric turbulence parameters is important for diagnostic and post-processing applications, such as the evaluation of the AO performance and point spread function (PSF) reconstruction. 
Several techniques, based on the spatial or temporal correlation (also known as covariance) of the measured slope of ShackHartmann wavefront sensors (SH-WFSs), have been proposed to retrieve the vertical profiles of $C_{N}^{2}(h), \mathcal{L}_{0}(h)$ and $\boldsymbol{v}(h)$ (Wilson 2002; Butterley, Wilson \& Sarazin 2006; Cortés et al. 2012; Martin et al. 2016), and some have already been implemented into onsky WFAO systems (Lardière et al. 2014; Neichel et al. 2014; Vidal et al. 2014).

The SLOpe Detection And Ranging (SLODAR) method is a method commonly used to estimate the vertical profile of $C_{N}^{2}$ in real time from measurements of multiple SH-WFSs. This method is based on optical triangulation between two or more stars, and it retrieves the vertical profile of $C_{N}^{2}$ from the intensities of peaks in the spatio cross-correlation deconvolved by the autocorrelation (Wilson 2002). We refer to this method as the deconvolved SLODAR. Its great advantage is that it is model-independent. In addition, using temporal cross-correlation of the measured slopes allows us to estimate the temporal features of the atmospheric turbulence (Wang, Schöck \& Chanan 2008; Guesalaga et al. 2014).

Another SLODAR approach proposed by Butterley et al. (2006) recovers the vertical profile of $C_{N}^{2}(h)$ by fitting the theoretical spatio cross-correlation to the observed spatial cross-correlation. This second approach is referred to as the fitted SLODAR in this paper. With the latter, unlike its predecessor, deconvolved SLODAR, we will be able to estimate the vertical profile of the $\mathcal{L}_{0}(h)$ by conforming to the von Karman power spectrum model for the theoretical spatio cross-correlation. If $\mathcal{L}_{0}$ has different values at different altitudes, then measuring the actual $\mathcal{L}_{0}$ value at each altitude is critical to obtain an accurate $C_{N}^{2}$ profile using the SLODAR method (Valenzuela et al. 2014).

The SLODAR methods using in situ real-time AO telemetry are thought to grasp more effects than off-site monitors, which cannot look at different objects along different lines of sight simultaneously with the AO observations. AO telemetry probes the atmosphere, the dome seeing and any instrument-specific aberrations, which can be disentangled with appropriate processing.

In this paper, we develop a new SLODAR method that minimizes fitting residuals over the autocorrelation and cross-correlation functions using analytical derivatives of the spatial phase structure function with respect to its parameters to accelerate the non-linear solver - in our case the Levenberg-Marquardt Algorithm (LMA). We then post-process on-sky telemetry from multiple SH-WFSs installed on RAVEN, a multi-object adaptive optics (MOAO) technical and science demonstrator on the Subaru telescope. The first SLODAR results using RAVEN have been reported by Lardière et al. (2014). Here, we improve our SLODAR method and provide consolidated statistics at Maunakea from a total of 12 nights in 2014 May and August and in 2015 June and July. To our knowledge, these are the first $C_{N}^{2}$ profiles reported for Maunakea based on the SLODAR method using an 8-m class telescope.

This paper is organized as follows. In Section 2, we review the RAVEN MOAO system and the on-sky observations of RAVEN on the Subaru telescope. Then, we present the theoretical model and non-linear minimization for our SLODAR method in Section 3. In Section 4, we describe the estimated statistics of the vertical profiles of $C_{N}^{2}$ and $\mathcal{L}_{0}$. Finally, we discuss our findings in Section 5 and we summarize the results in Section 6.

\section{RAVEN}

RAVEN is a MOAO technical and science demonstrator on the Subaru telescope at Maunakea, Hawaii. RAVEN is the first MOAO demonstrator on an 8-m class telescope. Details of the RAVEN system are summarized in Lardière et al. (2014). Here, we briefly review RAVEN and our on-sky observations.

RAVEN applies MOAO corrections simultaneously towards two science targets using a tomographic reconstruction. RAVEN has four open-loop (OL) SH-WFSs with $10 \times 10$ subapertures for three natural guide stars (NGSs) and one on-axis sodium laser guide star (LGS) installed on the Subaru telescope. Because of the obscuration by the telescope pupil, 72 subapertures in a SH-WFS are available, as shown in Fig. 2.

On-sky engineering and science observations with RAVEN were successfully completed with the Subaru telescope. We had 12 nights in total, spread over three runs in 2014 May and August and in 2015 June and July. Two science papers based on RAVEN data have already been published (Davidge et al. 2015; Lamb et al. 2016) and one has been accepted (Davidge et al. 2016). In total, $60 \mathrm{~h}$ on-sky telemetry from three OL-WFSs are recorded in the on-sky observations.

During the on-sky observations, we estimated the turbulence profile by using SLODAR with three NGS OL-WFSs for the tomographic reconstruction. The OL-WFSs were operated mostly with frame rates of $100-250 \mathrm{~Hz}$ depending on the brightness of the NGSs, which were brighter than $R<13.5$ mag in most cases. The spot positions for each subaperture were measured primarily with the threshold centre of gravity ( $\mathrm{tCoG})$ algorithm. We also tested a correlation centroiding method. The correlation centroiding provided slightly smaller measurement noise than the tCoG algorithm for guide stars brighter than $R<13.5$ mag; for guide stars fainter than $R>13.5 \mathrm{mag}$, the correlation centroiding gave much smaller measurement noise (Andersen et al. 2014). From this fact, we expect that the measurement noise is negligible when the guide star magnitude is brighter than $R<14$ mag because there is almost no difference in the centroiding accuracy of both methods.

The SLODAR method used for on-sky observations, which is referred to as the on-sky SLODAR, can measure only the vertical profiles of $C_{N}^{2}(h)$ and assumes a constant outer scale of $30 \mathrm{~m}$ over all altitudes. In addition, the on-sky SLODAR measures the turbulence up to $12 \mathrm{~km}$ to reduce the number of turbulence layers and to accelerate the computation of the tomographic reconstruction matrix. Although the on-sky SLODAR worked during the on-sky observations, this method is not enough to measure the complete turbulence profile.

We also tried to estimate wind speed and direction at each altitude during on-sky observations using a method presented in Ono et al. (2016), and we tested new reconstruction algorithms (Correia et al. 2015; Ono et al. 2016). However, in this paper, we concentrate on retrieving the vertical profiles of $C_{N}^{2}(h)$ and $\mathcal{L}_{0}(h)$.

\section{SLODAR}

In this section, we develop the SLODAR method by improving upon the initial formulations to include the estimation of a $\mathcal{L}_{0}(h)$ profile. We start by presenting our method for computing the spatial correlations from WFS data and then we show how to compute such functions analytically from von Karman spatial structure functions. The latter are then differentiated with respect to their parameters in order to compute the Jacobian as part of the non-linear optimization routine that will minimize a $\chi^{2}$ criterion, fitting data covariances to theoretical correlations. 


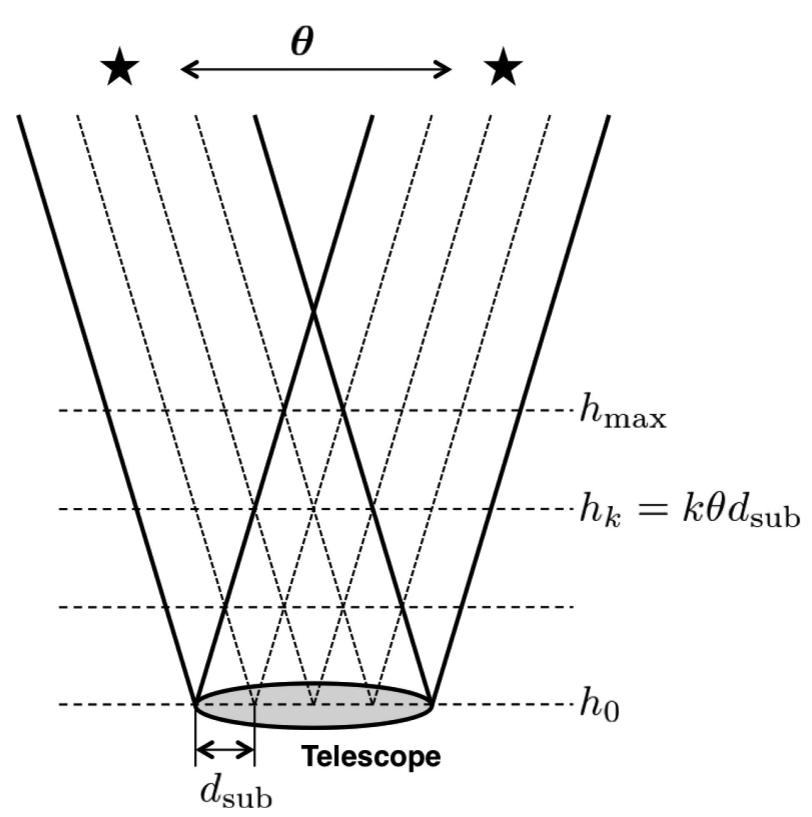

Figure 1. Schematic image of SLODAR with two stars separating $\boldsymbol{\theta}$.

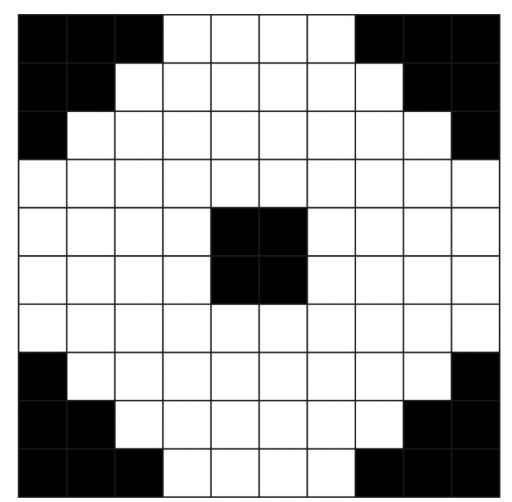

Figure 2. Valid subapertures of SH-WFS in RAVEN.

\subsection{Spatial correlations of slope data}

As mentioned previously, the SLODAR method estimates the turbulence profile via the triangulation between two stars, as shown Fig. 1. A turbulence layer at altitude $h$ makes a peak in the spatio cross-correlation with a spatial offset corresponding to $h \theta$, where $\theta$ is the angular separation of the two stars. The offset between the peaks in cross-correlation allow us to distinguish the atmospheric turbulence layers at different altitudes.

Throughout this paper, it is assumed that the difference in centroiding gain between different subapertures is negligible, which would be valid for NGS SH-WFSs. However, in the case with strong spot elongations, such as LGS-based systems in future ELTs, each subaperture will have a different centroiding gain, and this difference in the centroiding gain should be corrected before the following SLODAR process.

The $x$-slope of the subaperture indexed as $(i, j)$ of the $p$ th WFS is noted as $s_{i, j}^{x[p]}$. In order to remove the effect of overall tip/tilt caused by the telescope guiding error and wind shake and vibration from the telescope or/and instruments, the overall tip/tilt (i.e. the mean slope over all subapertures) should be subtracted from each frame before the correlation is computed.
The mean slope over all subapertures is given as

$\overline{s^{x[p]}}=\frac{1}{N_{\mathrm{sub}}} \sum_{i, j} s_{i, j}^{x[p]}$,

where $N_{\text {sub }}$ is the total number of valid subapertures in a SH-WFS. The spatio cross-correlation between the $x$-slopes of the $p$ th WFS and the $x$-slopes of the $q$ th WFS, with the subaperture offset of ( $\delta i$, $\delta j$ ) and the tip/tilt removal, is defined as

$$
C^{x[p] x[q]}(\delta i, \delta j)=\frac{\sum_{i, j}\left\langle\left(s_{i, j}^{x[p]}-\overline{s^{x[p]}}\right)\left(s_{i+\delta i, j+\delta j}^{x[q]}-\overline{s^{x[q]}}\right)\right\rangle}{O(\delta i, \delta j)} .
$$

Here, $\sum_{i, j}$ denotes a summation for all valid subapertures, \langle\rangle denotes the average over the time series and $O(\delta i, \delta j)$ denotes the number of the valid subaperture pairs with the offset of $(\delta i, \delta j)$, which is equal to the spatio autocorrelation of a pupil mask shown in Fig. 2. The autocorrelation for the $p$ th WFS, $A^{x[p]}$, can be computed by equation (2) taking $q=p$.

In equation (2), the cross-correlation is averaged for the same offset of $(\delta i, \delta j)$ by $O(\delta i, \delta j)$. This process makes the size of the correlation map small, and reduces the computational burden of SLODAR. In the case of RAVEN, the size of averaged and nonaveraged correlation maps is $19 \times 19$ and $100 \times 100$, respectively. This difference becomes critical for future ELTs, which have more than five WFSs and $\sim 60 \times 60$ subapertures. Furthermore, the averaging process can make the signal-to-noise ratio $(\mathrm{S} / \mathrm{N})$ high.

\subsection{Theoretical model of spatial correlation}

The SH-WFS measures a phase gradient averaged over a subaperture; it is modelled as

$s_{i, j}^{x[p]}=\int_{-d_{\text {sub }} / 2}^{d_{\text {sub }} / 2} \int_{-d_{\text {sub } / 2}}^{d_{\text {sub }} / 2} \frac{\partial \phi(x, y)}{\partial x} \mathrm{~d} x \mathrm{~d} y / S_{\text {sub }}$.

We assume that all subapertures are square, $(x, y)$ is a spatial coordinate with its origin at the centre of subaperture, $\phi(x, y)$ is a phase value at $(x, y)$ and $S_{\text {sub }}=d_{\text {sub }}^{2}$. Here, we use the approximated model for the SH-WFS slope to accelerate the computation presented in Martin et al. (2012). In the approximation, the slopes are considered as the phase difference between two midpoints on both sides of the subapertures, thus permitting the removal of the integrations from equation (3) and yielding

$s_{i, j}^{x[p]} \approx \frac{1}{d_{\text {sub }}}\left[\phi\left(\frac{d_{\text {sub }}}{2}, 0\right)-\phi\left(-\frac{d_{\text {sub }}}{2}, 0\right)\right]$.

We consider here a cross-correlation caused by single atmospheric layer at altitude $h$. In this case, the spatial distance $h \boldsymbol{\theta}$ between the projected pupils of two stars at altitude $h$ should be taken into account in the theoretical expression. We start from equation (4) and use the definition of the phase structure function $D_{\phi}(\boldsymbol{\rho})=\left\langle[\phi(\boldsymbol{r})-\phi(\boldsymbol{r}+\boldsymbol{\rho})]^{2}\right\rangle$ and the equality $2(A-a)(B-$ $b)=-(A-B)^{2}+(A-b)^{2}+(a-B)^{2}-(a-b)^{2}$. With these definitions, the slope correlation corresponding to an atmospheric turbulence layer at altitude $h$ can be given as

$$
\begin{aligned}
\left\langle s_{i, j}^{x[p]} s_{i+\delta i, j+\delta j}^{x[q]}\right\rangle= & \frac{1}{2 d_{\text {sub }}^{2}}\left[-2 D_{\phi}\left(\boldsymbol{\Delta}_{\boldsymbol{h}}\right)+D_{\phi}\left(-d_{\mathrm{sub}} \boldsymbol{u}_{\boldsymbol{x}}+\boldsymbol{\Delta}_{\boldsymbol{h}}\right)\right. \\
& \left.+D_{\phi}\left(d_{\mathrm{sub}} \boldsymbol{u}_{\boldsymbol{x}}+\boldsymbol{\Delta}_{\boldsymbol{h}}\right)\right],
\end{aligned}
$$

where $\boldsymbol{u}_{\boldsymbol{x}}$ is a unit vector in the $x$-direction, $\boldsymbol{\Delta}_{\boldsymbol{h}}=\left(\Delta_{x}, \Delta_{x}\right)$ is the projected distance at $h$ between the centre of the $(i, j)$ subaperture in the $p$ th WFS and the $(i+\delta i, j+\delta j)$ subaperture in the $q$ th WFS, 
and $\Delta_{x}=d_{\text {sub }} \delta i+h \theta_{x}$ and $\Delta_{y}=d_{\text {sub }} \delta j+h \theta_{y}$. The spatial phase structure function for the von Karman power spectrum is given as

$$
\begin{aligned}
D_{\phi}(\rho)= & 0.17253\left(\frac{L_{0}}{r_{0}}\right)^{5 / 3} \\
& \times\left[1-\frac{2^{1 / 6}}{\Gamma(5 / 6)}\left(\frac{2 \pi \rho}{L_{0}}\right)^{5 / 6} K_{5 / 6}\left(\frac{2 \pi \rho}{L_{0}}\right)\right],
\end{aligned}
$$

where $K$ represents the modified Bessel function of the second kind.

The removal of the overall tip/tilt should also be considered for the reasons discussed previously. The theoretical correlation with the tip/tilt removal is given as

$$
\begin{gathered}
\left\langle\left(s_{i, j}^{x[p]}-\overline{s^{x[p]}}\right)\left(s_{i+\delta i, j+\delta j}^{x[q]}-\overline{s^{x[q]}}\right)\right\rangle \\
=\left\langle s_{i, j}^{x[p]} s_{i+\delta i, j+\delta j}^{x[q]}\right\rangle-\left\langle\overline{s^{x[p]}} s_{i+\delta i, j+\delta j}^{x[q]}\right\rangle \\
-\left\langle s_{i, j}^{x[p]} \overline{s^{x[q]}}\right\rangle+\left\langle\overline{s^{x[p]}} \overline{s^{x[q]}}\right\rangle,
\end{gathered}
$$

where

$$
\left\langle\overline{s^{x[p]}} \overline{s^{x[q]}}\right\rangle=\frac{1}{N_{\text {sub }}^{2}} \sum_{i^{\prime}, j^{\prime}} \sum_{i, j}\left\langle s_{i^{\prime}, j^{\prime}}^{x[p]} s_{i, j}^{x[q]}\right\rangle
$$

and

$$
\left\langle\overline{s^{x[p]}} s_{i+\delta i, j+\delta j}^{x[q]}\right\rangle=\frac{1}{N_{\text {sub }}} \sum_{i^{\prime}, j^{\prime}}\left\langle s_{i^{\prime}, j^{\prime}}^{x[p]} s_{i+\delta i, j+\delta j}^{x[q]}\right\rangle .
$$

Equations (8) and (9) show the autocorrelation of the mean slope and the cross-correlation between the mean slope and each slope, respectively.

All theoretical correlations in equations (7), (8) and (9) can be computed using equation (5). Then, the final expression of the tip/tilt removed theoretical correlation is given by substituting equation (7) into equation (2). The $y-y, x-y$ and $y-x$ slope correlations can be given in a similar way, but in this work the $x-y$ and $y-x$ slope correlations are not used for turbulence profile estimation, because they have only weak correlation compared to the $x-x$ and $y-y$ slope correlations.

The theoretical model for the autocorrelation is given in a similar way to the cross-correlation as $q=p$. In the case of NGSs, the theoretical autocorrelation depends only on $C_{N}^{2}\left(h_{k}\right)$ and $L_{0}\left(h_{k}\right)$ and not on the altitude. However, in the case of LGSs, the theoretical autocorrelation depends on the altitude because the projected pupil size becomes smaller with altitude because of the cone effect.

\subsection{Model fitting}

The vertical profiles of $C_{N}^{2}(h)$ and $\mathcal{L}_{0}(h)$ can be recovered by fitting the theoretical correlations to the observed correlation. Here, we define a vector $\boldsymbol{C}^{x[p q]}$ as a concatenation of $C^{x[p] x[q]}(\delta i, \delta j)$ for all subaperture offsets $(\delta i, \delta j)$. The observed cross-correlation is noted as $\boldsymbol{C}_{\mathrm{obs}}$, and the theoretical cross-correlation of the $k$ th altitude bin is denoted as $\boldsymbol{C}_{\text {theo, } k}$. When only the $C_{N}^{2}$ profile is estimated, the profile can be obtained by a linear fit (Butterley et al. 2006; Cortés et al. 2012). However, the fit becomes more complex when $\mathcal{L}_{0}(h)$ are also estimated, because $\mathcal{L}_{0}(h)$ are non-linear parameters in the theoretical model.

The left panel of Fig. 3 shows an example of the observed crosscorrelation computed from $1 \mathrm{~min}$ of on-sky telemetry data taken by RAVEN. You can see the two correlation peaks on the baseline of two NGSs, shown as the dashed line. The central peak corresponds to the ground layer and the other peak corresponds to a high altitude

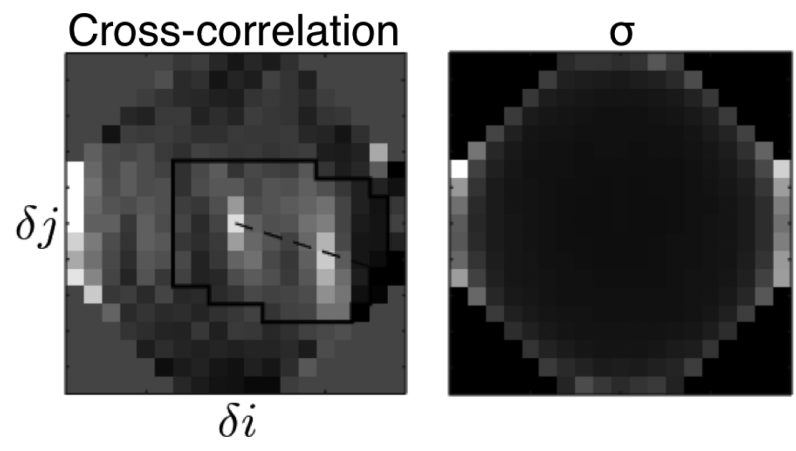

Figure 3. Examples of the observed cross-correlation (left) and standard deviation of the cross-correlation (right) computed from the on-sky telemetry data taken by RAVEN. The dashed line in the left panel shows the baseline of two stars. The area surrounded by the black line is used for the fitting.

layer. The edge of the cross-correlation map is very noisy because of the small number of subaperture pairs with large $(\delta i, \delta j)$. A similar trend can be seen in the standard deviation of the cross-correlation over 1 min shown in the right panel of Fig. 3. In order to reduce the effect from the noisy correlation values resulting from the small number of subaperture pairs, the temporal standard deviation map is used as the weight of the fit, the cross-correlation values with fewer than five subaperture pairs are removed from the fitting and only the correlation values around the baseline are used in the fitting, which has a high $\mathrm{S} / \mathrm{N}$, shown as the area in the black line in Fig. 3 (Cortés et al. 2012). It should be noted that, as the outer scale affects the shape of the correlation peak, the wide area around the baseline should be selected to estimate $\mathcal{L}_{0}(h)$ at each altitude.

The altitude is input in our SLODAR fitting. The altitude resolution $\delta h$ is given by $\delta h=d / \theta$ when the baseline of two stars is aligned to the $x$ - or $y$-axis of a WFS lenslet array. However, when the baseline is not aligned to the $x$ - or $y$-axis of the lenslet array, as in Fig. 3, the optimal resolution is given as $\delta h=d /(\theta \sin \alpha)$, where $\alpha$ is the angle of the baseline with respect to the $x$-axis for $\theta_{x}>\theta_{y}$ or the $y$-axis for $\theta_{x}<\theta_{y}$. In the case of RAVEN, the optimal $\delta h$ depends on the NGSs asterism and changes with time as a result of the field rotation, and thus it should be updated during the observation. The maximum altitude $h_{\max }$, which can be sensed via the triangulation using the cross-correlation, also varies.

Because RAVEN uses three NGSs, the SLODAR method can be computed with three different NGS pairs. Each pair has a different $\delta h$ and $h_{\max }$, and the pair with narrow angular separation provides a small $\delta h$ and high $h_{\max }$, whereas wide separation gives a large $\delta h$ and low $h_{\max }$. In order to deal with all pairs together, we use the minimum altitude resolution in three pairs for all three NGS pairs. Although it causes oversampling of altitude for the other two pairs with larger $\delta h$, we have more measurements from three pairs to derive the turbulence profile. The maximum altitude is determined by the GS pair with the smallest angular separation in the three pairs. Fig. 4 shows histograms of $\delta h$ (the top panel) and $h_{\max }$ (the bottom panel) during the on-sky observations. The range of the altitude resolution is from 0.75 to $3 \mathrm{~km}$ and the median value is $1.5 \mathrm{~km}$. The maximum altitude ranges over a wide range and, in some cases, turbulence only up to $5 \mathrm{~km}$ can be sensed by the cross-correlation depending on the NGS configuration.

The turbulence above $h_{\max }$ cannot be sensed via the triangulation with the cross-correlations (hereafter referred to as unsensed turbulence), but it can be measured by the autocorrelation of measurements from NGSs. In this paper, we use both the autocorrelations and cross-correlations simultaneously to estimate the integrated $C_{N}^{2}$ 

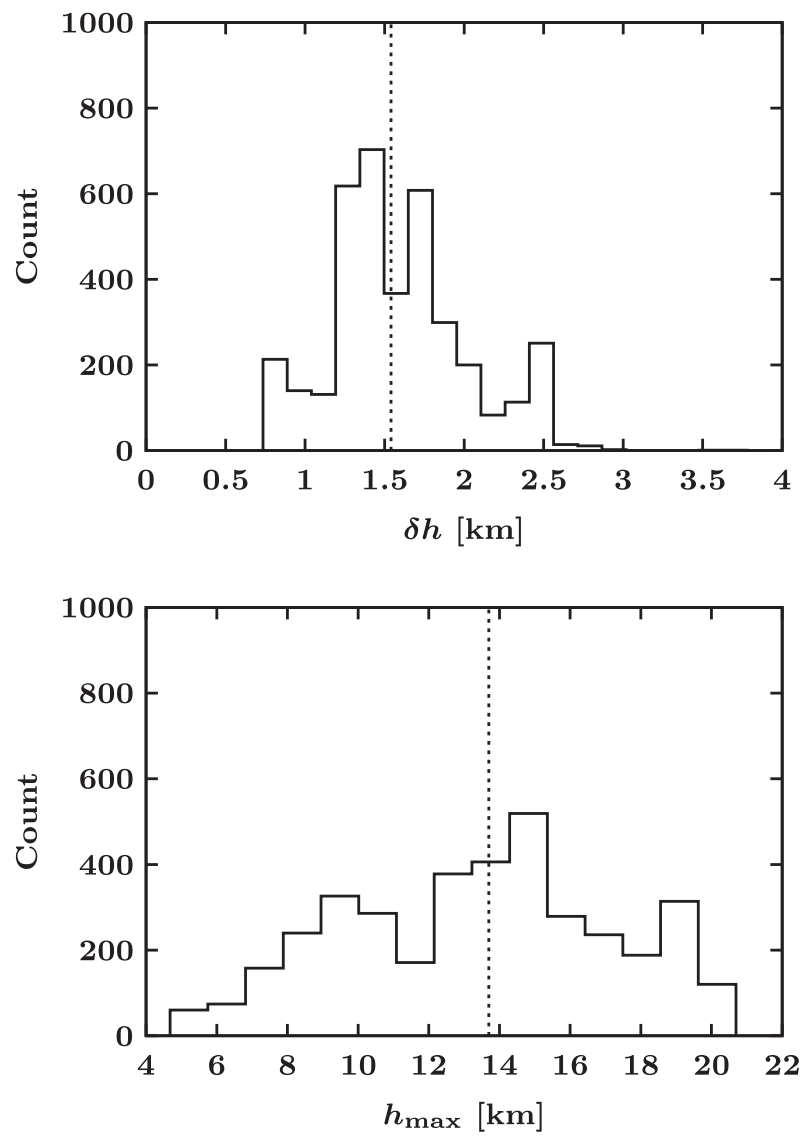

Figure 4. Histograms of the altitude resolution $\delta h$ (top) and the maximum altitude $h_{\max }$ (bottom) of SLODAR during all on-sky observations. The vertical dotted lines show the median value, which is $1.5 \mathrm{~km}$ for $\delta h$ and $13.7 \mathrm{~km}$ for $h_{\max }$. Here, $\delta h$ and $h_{\max }$ are scaled for the zenith direction.

and $\mathcal{L}_{0}$ of the unsensed turbulence. It should be noted that this method cannot estimate the altitudes of the unsensed turbulence.

The $\chi^{2}$ value to be minimized in the fitting process is given as

$$
\begin{aligned}
\chi^{2}= & \| \sum_{p, q} \boldsymbol{W}_{\mathbf{c}}^{x[p q]}\left(\boldsymbol{C}_{\mathrm{obs}}^{x[p q]}-\sum_{k}^{N_{\text {layer }}} \boldsymbol{C}_{\text {theo }, k}^{x[p q]}\right) \\
& +\sum_{p, q} \boldsymbol{W}_{\mathbf{c}}{ }^{y[p q]}\left(\boldsymbol{C}_{\mathrm{obs}}^{y[p q]}-\sum_{k}^{N_{\text {layer }}} \boldsymbol{C}_{\text {theo }, k}^{y[p q]}\right) \\
& +\sum_{p} \boldsymbol{W}_{\mathrm{a}}{ }^{x[p]}\left[\boldsymbol{A}_{\mathrm{obs}}^{x[p]}-\left(\sum_{k}^{N_{\text {layer }}} \boldsymbol{A}_{\text {theo }, k}^{x[p]}+\boldsymbol{A}_{\text {theo, }, \infty}^{x[p]}\right)\right] \\
& +\sum_{p} \boldsymbol{W}_{\mathrm{a}}{ }^{y[p]}\left[\boldsymbol{A}_{\mathrm{obs}}^{y[p]}-\left(\sum_{k}^{N_{\text {layer }}} \boldsymbol{A}_{\text {theo, }, k}^{y[p]}+\boldsymbol{A}_{\text {theo, }, \infty}^{y[p]}\right)\right] \|^{2} .
\end{aligned}
$$

Here, $N_{\text {layer }}$ is the number of altitude bins and $\boldsymbol{W}$ represents the weighting matrix, which extracts the correlation values used in the fitting according to the criteria mentioned previously and a weight for each correlation value by a square inverse of the standard deviation of correlation over a time series. It is known that the central correlation value of the autocorrelation is enhanced by a correlation of measurement noise in the slope (Butterley et al. 2006), and so we remove it from the fitting using $\boldsymbol{W}_{\mathrm{a}}$. The theoretical correlation is a function of altitude $h_{k}$, Fried parameter $r_{0, k}$ and outer scale $\mathcal{L}_{0, k}$; that is, $\boldsymbol{C}_{\text {theo, }, k}=\boldsymbol{C}_{\text {theo }}\left(h_{k}, r_{0, k}, \mathcal{L}_{0, k}\right)$, where $r_{0, k}$ relates to $C_{N}^{2}$ as

$r_{0, k}=\left[0.423 k^{2} C_{N}^{2}\left(h_{k}\right)\right]^{-3 / 5}$,

where $k=2 \pi / \lambda$ and $\lambda$ is a wavelength.

We add the theoretical model of the unsensed turbulence, $\boldsymbol{A}_{\text {theo, } \infty}$, only to the autocorrelation fitting term to estimate $C_{N}^{2}$ and $\mathcal{L}_{0}$ of the unsensed turbulence, which does not affect the cross-correlation. So, the free parameters in equation (10) are $r_{0}$ (or $C_{N}^{2}$ ) and $\mathcal{L}_{0}$ of $N_{\text {layer }}$ altitude bins and the unsensed turbulence; we have $2 \times N_{\text {layer }}$ +2 free parameters for the fitting.

Equation (10) is a non-linear least-squares problem and can be simply expressed as $\chi^{2}=\sum_{i}\left[y_{i}-f\left(x_{i}, \boldsymbol{\beta}\right)\right]^{2} / \sigma_{i}^{2}$, where $y$ is a measured value, $f$ is a model to be fitted, $\boldsymbol{\beta}$ represents parameters to be estimated and $1 / \sigma^{2}$ is a weight. We use the LMA to determine the best parameters, which minimizes $\chi^{2}$. The LMA finds the best parameters iteratively and, in each iteration, the parameter $\boldsymbol{\beta}$ is updated to $\boldsymbol{\beta}+\boldsymbol{\delta} \boldsymbol{\beta}$. In order to compute $\boldsymbol{\delta} \boldsymbol{\beta}$, the model function $f$ is approximated as $f\left(x_{i}, \boldsymbol{\beta}+\boldsymbol{\delta} \boldsymbol{\beta}\right) \approx f\left(x_{i}, \boldsymbol{\beta}\right)+\boldsymbol{J} \boldsymbol{\delta} \boldsymbol{\beta}$, where $\boldsymbol{J}$ is the Jacobian matrix and each element of $\boldsymbol{J}$ is a partial deviation of $f$ with respect to $\boldsymbol{\beta}$, and thus $J_{i j}=\partial f\left(x_{i}, \boldsymbol{\beta}\right) / \partial \beta_{j}$. In our case, $f$ consists of the theoretical correlations, and to compute the Jacobian matrix we need a partial deviation of the von Karman structure function in equation (6) with respect to $r_{0}$ and $\mathcal{L}_{0}$. In the Appendix, we present the computation. In the iteration of the LMA, we add a condition that $r_{0}$ and $\mathcal{L}_{0}$ at all altitudes are larger than zero. In addition, an upper limit for $\mathcal{L}_{0}$ is set to $100 \mathrm{~m}$.

It should be noted that this method can be applied to a case in which the altitudes of turbulence layers are free parameters and are also applied to the temporal correlation to estimate the wind speed and direction at each altitude. In the theoretical model, altitude, wind speed and direction affect the distance between two subapertures (i.e., $\rho$ in equation 6). For example, the partial deviation of equation (6) with respect to the altitude can be given as $\partial D_{\phi} / \partial h=(\partial \rho / \partial h)\left(\partial D_{\phi} / \partial \rho\right)$. The term of $\partial D_{\phi} / \partial \rho$ can be computed in a similar way to $\partial D_{\phi} / \partial \mathcal{L}_{0}$ as shown in the Appendix. The computation of $\partial \rho / \partial h$ depends on the model (Martin et al. 2016), but it is usually not complex.

\section{RESULTS}

\subsection{Statistics of the atmospheric turbulence at Maunakea}

Here, we present the statistics of the atmospheric turbulence at Maunakea measured from the RAVEN on-sky telemetry data by our SLODAR method. Fig. 5 shows histograms of seeing (top), $C_{N}^{2}$ (middle) and $\mathcal{L}_{0}$ (bottom) for the total turbulence, the ground turbulence $(0 \leq h<1.5 \mathrm{~km})$, the upper turbulence $(h \geq 1.5 \mathrm{~km}$, including the unsensed turbulence) and the unsensed turbulence. It is noted that our ground layer includes the turbulence up to $1.5 \mathrm{~km}$ and therefore it is not the pure ground layer. The seeing is computed from the estimated $r_{0}$ via the equation Seeing $=0.98 \lambda / r_{0}$. The integrated $\mathcal{L}_{0}$ over an altitude range is usually defined as

$\mathcal{L}_{0}\left(h_{1}<h<h_{2}\right)=\left[\frac{\int_{h_{1}}^{h_{2}} \mathcal{L}_{0}^{5 / 3}(h) C_{N}^{2}(h) \mathrm{d} h}{\int_{h_{1}}^{h_{2}} C_{N}^{2}(h) \mathrm{d} h}\right]^{3 / 5}$.

In this paper, seeing and $r_{0}$ are defined at $\lambda=500 \mathrm{~nm}$. Also, these values are scaled for the zenith direction when it is not stated.

The median value of the total seeing during the RAVEN observations is 0.460 arcsec, and this is a good condition compared with the results from the literature (0.602 arcsec in Els et al. 2009; 0.684 arcsec in Miyashita et al. (2004)). The reason why we measure 

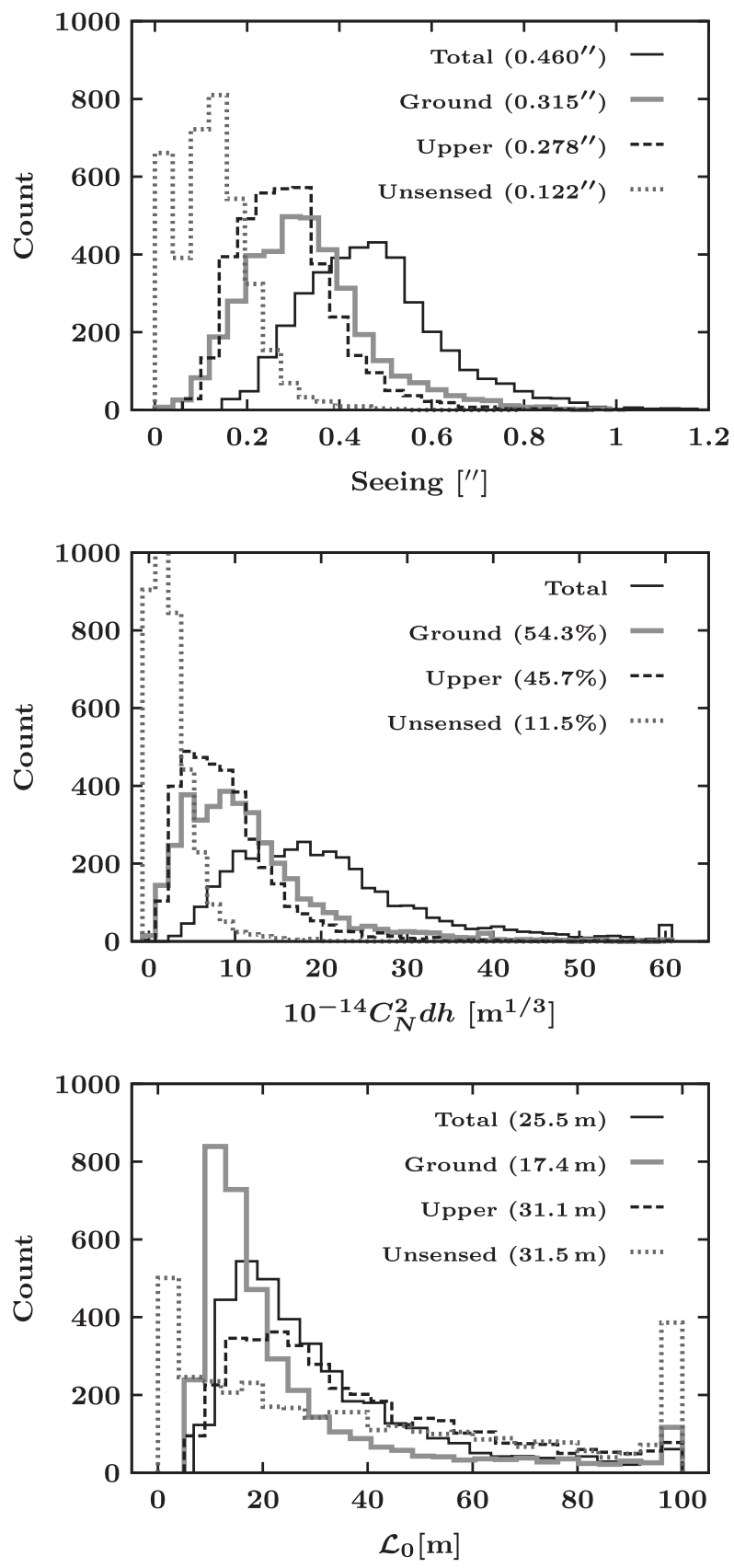

Figure 5. Histograms of seeing (top), $C_{N}^{2}$ (middle) and $\mathcal{L}_{0}$ (bottom) for the total turbulence (black solid lines), the ground turbulence (grey bold lines), upper turbulence (black dashed lines) and the unsensed turbulence (grey dotted lines). The values in parentheses show median values for seeing, $C_{N}^{2}$ fraction and $\mathcal{L}_{0}$. The seeing is scaled for the zenith direction.

such good seeing is that the on-sky observations were performed mostly in May, June and July, when the seeing is better than in other seasons (Miyashita et al. 2004).

As expected, the ground layer has the largest contribution and the median value of the $C_{N}^{2}$ fraction of the ground layer is 54.3 per cent. Such a dominant ground layer at Maunakea has been reported in previous results based on different methods: 85 per cent based on the multi-aperture scintillation sensor (MASS) and the differential image motion monitor (DIMM) for the Thirty Meter Telescope (TMT) site testing in Els et al. (2009), and 54 per cent based on MASS-DIMM and 40 per cent based on SCIntillation

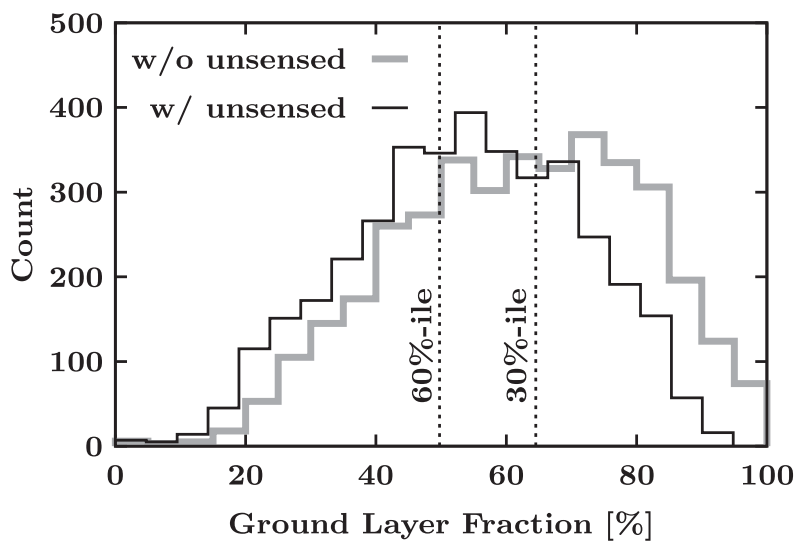

Figure 6. Histogram of the $C_{N}^{2}$ fraction of the ground layer $(h<1.5 \mathrm{~km})$. The black line shows the histogram including the unsensed turbulence and the vertical dotted lines present the ground layer fraction of the 60 and 30 percentiles, which corresponds to the ground layer fractions of 50 and 64.5 per cent, respectively. The grey bold line shows the histogram without the unsensed turbulence.

Detection And Ranging (SCIDAR) in Tokovinin et al. (2005). In addition, the large contribution of the ground layer can also be seen in Fig. 6, which shows a histogram of the $C_{N}^{2}$ fraction of the ground layer. From this figure, the probability that more than 50 per cent of the turbulence is included in the ground layer (up to $1.5 \mathrm{~km}$ ) is 60 per cent.

Fig. 5 represents the existence of the unsensed turbulence above the maximum altitude to which the SLODAR is sensitive. In the middle panel of Fig. 5, the $C_{N}^{2}$ histogram of the unsensed turbulence (shown as the dotted grey line) has a narrow peak, which is shown to be a relatively small contribution with a median value of 11.5 per cent of the total. However, if we do not consider the unsensed turbulence, it is possible that the fraction of the ground layer is overestimated, as shown in Fig. 6. We return to this discussion about the unsensed turbulence later in the paper.

The histograms of the outer scale have a steep slope at small $\mathcal{L}_{0}$ and a long tail at large $\mathcal{L}_{0}$. This is because the SLODAR method is less sensitive to $\mathcal{L}_{0}$ values larger than the telescope aperture, which affects the tip/tilt modes over the aperture, and we have a large uncertainty for large $\mathcal{L}_{0}$. The median integrated outer scale is $25.5 \mathrm{~m}$, consistent with a previous result at Maunakea $(26.9 \mathrm{~m}$ in Maire et al. 2007). However, it is reported that the estimation of $\mathcal{L}_{0}$ by SLODAR is biased to two to three times the telescope aperture because of the weak sensitivity of SLODAR to large $\mathcal{L}_{0}$ (Martin et al. 2016); our result is close to the size of three times the telescope aperture. However, similar or smaller values $\left(\mathcal{L}_{0}=10-20 \mathrm{~m}\right)$ have been found based on instruments with various spatial scales (1$100 \mathrm{~m}$ ) at a different site (Ziad et al. 2004). There are no definite results yet on the actual values of $\mathcal{L}_{0}$, and more measurements with various instruments are required. The trend for the largest outer scales to be at higher altitudes, as shown in the histogram, has been reported at several observation sites (Maire et al. 2007; Guesalaga et al. 2016; Martin et al. 2016). One possible explanation of this is that the SLODAR method is more sensitive to large $\mathcal{L}_{0}$ at high altitudes, because of the large meta-pupil at high altitudes.

\subsection{Median profiles}

Fig. 7 and Table 1 present the mean profile of $C_{N}^{2}$ and the median profile of $\mathcal{L}_{0}$. In order to compare our estimates with results from 

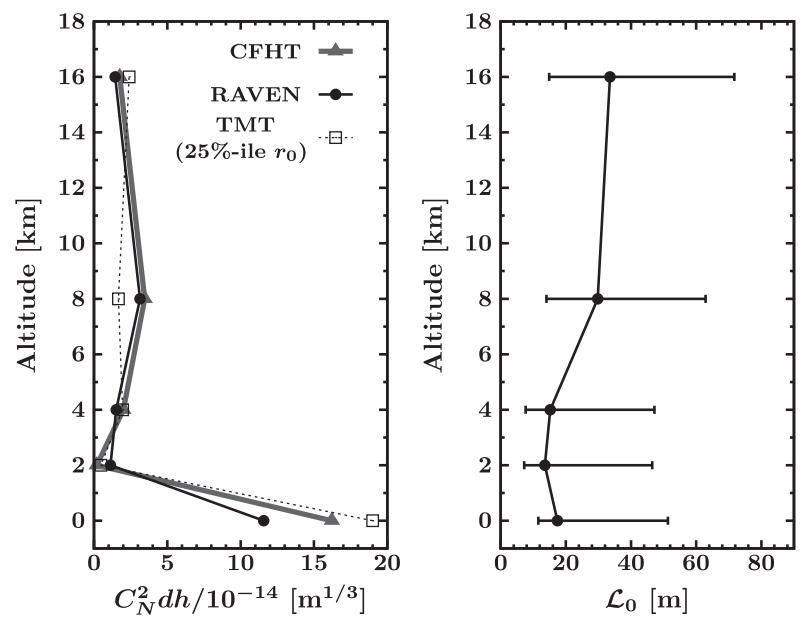

Figure 7. Left: mean $C_{N}^{2}$ vertical profile from the RAVEN SLODAR (black circles and solid line), the CFHT MASS and DIMM (grey triangles and bold line) and TMT site characterization with 25 percentile $r_{0}$ (black squares and dotted line). Right: median outer-scale profile from the RAVEN SLODAR. The error bars are computed from standard deviations below and above the median $\mathcal{L}_{0}$. The values of $C_{N}^{2}$ and the outer scale in the profiles are listed in Table 1.

other instruments, the estimated profiles are resampled into five altitude bins: ground layer $(0 \leq h \leq 1.5 \mathrm{~km}), 2 \mathrm{~km}(1.5<h \leq 3 \mathrm{~km})$, $4 \mathrm{~km}(3<h \leq 6 \mathrm{~km}), 8 \mathrm{~km}(6<h \leq 12 \mathrm{~km})$ and $16 \mathrm{~km}(12<h)$. In the left panel of the figure, as mentioned previously, the ground layer has a strong contribution of $C_{N}^{2}$. There is a weak turbulence at $8 \mathrm{~km}$. At $2 \mathrm{~km}$, turbulence is not detected most of the time.

For comparison, we plot the mean $C_{N}^{2}$ profiles at Maunakea from a MASS and a DIMM at the Canada-France-Hawaii Telescope (CFHT; hereafter referred to as the CFHT profile; available in http://mkwc.ifa.hawaii.edu/current/seeing/) and from the site characterization over $\sim 2.5 \mathrm{yr}$ for the TMT in Els et al. (2009) (referred to as the TMT profile). The DIMM at the CFHT monitored the total seeing on nine nights out of 12 nights of the RAVEN observations. The MASS measured the vertical profiles of $C_{N}^{2}$ at altitudes higher than $0.5 \mathrm{~km}$ on seven nights out of our observations. The ground layer of the CFHT profile is estimated from the difference between the total seeing from the DIMM and the MASS. As the MASS and DIMM are not synchronized, the data overlapping each other within $\pm 60 \mathrm{~s}$ are used for the ground layer.

The mean $C_{N}^{2}$ profile from the SLODAR is in good agreement with the CFHT profile, except for the ground layer, despite the different locations of the Subaru telescope and the CHFT. This means that the turbulence at high altitudes is common over a wide range of sky, but the ground layer depends on the location of the monitoring telescope. In addition, the dome seeing of Subaru also affects this difference in the ground layer. For the 2-km bin, the mean $C_{N}^{2}$ from the SLODAR is larger than the value from the CFHT. This is because the altitude resolution of the SLODAR is not enough to resolve the turbulence around the 2-km bin and there is contaminated turbulence from other altitude bins through the resampling process.

For the TMT profile, we selected a profile with good seeing conditions ( 25 percentile $r_{0}$; seeing $<0.55$ arcsec), because our results correspond to good seeing conditions (our mean seeing was 0.46 arcsec). Similar to the CFHT profile, there is a large difference in the ground layer between the RAVEN and the TMT profiles, and the weak ground layer in the RAVEN profile results in good seeing conditions during the RAVEN observations. The trend at high altitudes is different between the RAVEN and TMT profiles.

In the right panel of Fig. 7, the outer scale is larger at higher altitudes. The error bars of $\mathcal{L}_{0}$ show the standard deviations below and above the median $\mathcal{L}_{0}$, and the outer-scale spread over a wide range as $\mathcal{L}_{0}$ is larger than the median value. These findings are consistent with the results in Section 4.1 and Fig. 5.

\section{DISCUSSION}

\subsection{Comparison with CFHT DIMM and MASS}

In Section 4, we compared the mean $C_{N}^{2}$ profile estimated by our SLODAR with that from the CFHT MASS-DIMM, and there is a good general agreement except for the ground layer. In this section, we discuss this comparison in more detail. However, it should be noted that the CFHT MASS-DIMM cannot be perfectly compared to our SLODAR because these instruments have different altitude resolutions and observe different directions; moreover, Subaru and CFHT are located at different places atop Maunakea.

Fig. 8 shows the comparison of $C_{N}^{2}$ from the SLODAR and the MASS-DIMM at each altitude bin used in Fig. 7. A comparison of the total $C_{N}^{2}$ and $C_{N}^{2}$ at high altitudes $(h>1.5 \mathrm{~km})$ is also shown in the figure. In Fig. 8, the turbulence weaker than $C_{N}^{2} \mathrm{~d} h=10^{-16} \mathrm{~m}^{1 / 3}$ is not plotted because such a weak turbulence is affected by the measurement noise and is difficult to compare.

In Fig. 8(a), the total $C_{N}^{2}$ estimated by the SLODAR correlates with the total $C_{N}^{2}$ estimated by the DIMM. In Fig. 8(b), the ground layer also correlates but has larger scatter $(\mathrm{rms}=0.29 \mathrm{dex})$ than that of the total $C_{N}^{2}$ relation $(\mathrm{rms}=0.18 \mathrm{dex}$ ) in Fig. 8(a). The larger scatter of the ground turbulence might be affected by contamination from other altitude bins because of insufficient SLODAR altitude resolution, as mentioned in Section 4.2. The ground layer in the CFHT profile tends to be slightly larger than the

Table 1. Mean values of $C_{N}^{2}$ and median values of $\mathcal{L}_{0}$ at each altitude bin estimated by the RAVEN SLODAR. As comparison for the $C_{N}^{2}$ profile, mean $C_{N}^{2}$ profiles from the CFHT MASS-DIMM and the TMT site characterization in Els et al. (2009) are also listed. For $\mathcal{L}_{0}, \sigma_{\text {below }}$ and $\sigma_{\text {above }}$ represent the standard deviation computed below and above the median $\mathcal{L}_{0}$.

\begin{tabular}{lccccccc}
\hline Altitude $(\mathrm{km})$ & \multirow{2}{*}{ Range $(\mathrm{km})$} & \multicolumn{2}{c}{ Mean $C_{N}^{2} \mathrm{~d} h\left(10^{-14} \mathrm{~m}^{1 / 3}\right)$} & \multicolumn{3}{c}{$\mathcal{L}_{0}(\mathrm{~m})$} \\
& & RAVEN & CFHT & TMT & Median & $\sigma_{\text {below }}$ & $\sigma_{\text {above }}$ \\
\hline 0 & $0 \leq h \leq 1.5$ & 11.58 & 16.22 & 19.00 & 17.40 & 5.82 & 33.93 \\
2 & $1.5<h \leq 3$ & 1.12 & 0.19 & 0.48 & 13.57 & 6.34 & 32.96 \\
4 & $3<h \leq 6$ & 1.52 & 2.00 & 1.95 & 15.19 & 7.53 & 32.00 \\
8 & $6<h \leq 12$ & 3.14 & 3.46 & 1.67 & 29.76 & 15.70 & 33.13 \\
16 & $12<h$ & 1.45 & 1.75 & 2.40 & 33.54 & 18.66 & 38.15 \\
\hline
\end{tabular}




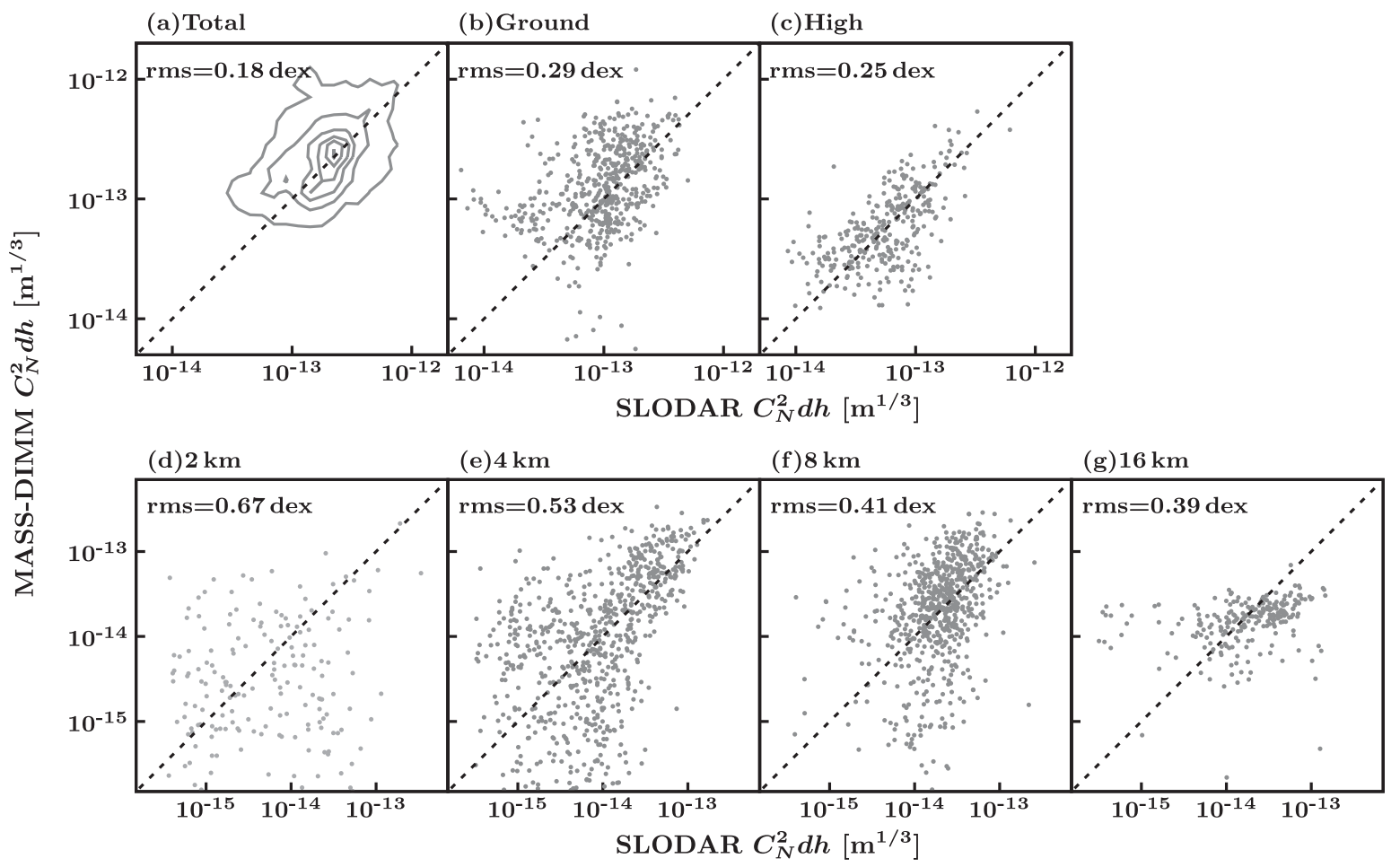

Figure 8. Comparison of the estimated $C_{N}^{2}$ between the RAVEN SLODAR and the CFHT MASS-DIMM. (a) Total $C_{N}^{2}$ from the SLODAR and the DIMM. (b) Ground layer; for the CFHT profile, the ground layer is computed from the MASS and the DIMM data. (d) $-(\mathrm{g}) C_{N}^{2}$ at five altitude bins used in Section 4.2 . For panel (a), we plot contours because the data points are too crowded to evaluate a correlation. In all panels, the turbulence weaker than $C_{N}^{2} \mathrm{~d} h=10^{-16} \mathrm{~m}^{1 / 3}$ is excluded because it is too weak to compare. The scatter rms value from the one-to-one relation (black dashed lines) are presented at the upper left of each panel in units of dex.

values in the SLODAR profile, as shown in the mean $C_{N}^{2}$ values in Section 4.1. In addition, the ground layer is affected by the dome seeing.

The $C_{N}^{2}$ values at high altitude in Fig. 8(c) show good agreement. However, the $C_{N}^{2}$ relation between the SLODAR and the MASS at each altitude bin, shown in Figs 8(d)-(g), shows a poorer correlation compared to the correlation of all the high altitudes in Fig. 8(c). One reason the correlation drops for each altitude bin is the contamination from other altitude bins and this has a large impact at $2 \mathrm{~km}$ in Fig. 8(d). Also, at $2 \mathrm{~km}$, turbulence weaker than $C_{N}^{2}=10^{-16} \mathrm{~m}^{1 / 3}$ is mostly detected, which is not included in the figure. At 4 and $8 \mathrm{~km}$, we see good correlations between $C_{N}^{2}$ from the SLODAR and the MASS for $C_{N}^{2}>10^{-14} \mathrm{~m}^{1 / 3}$, but there is a large dispersion for $C_{N}^{2}<10^{-14} \mathrm{~m}^{1 / 3}$ because of the contamination from other altitude bins. If we exclude turbulence weaker than $C_{N}^{2}<10^{-14}$ from the rms computation, the scatter rms values are 0.25 and 0.26 dex for 4 and $8 \mathrm{~km}$ bins, respectively, which is much smaller than the rms values including all data points. In the highest altitude bin at $16 \mathrm{~km}$, the scatter rms of $C_{N}^{2}$ is smaller than the values for 4 and $8 \mathrm{~km}$, but the number of data points is small because the maximum altitude of the SLODAR is sometimes limited to below $12 \mathrm{~km}$, depending on the GS configuration.

Considering the large difference at the ground layer between the SLODAR and the MASS-DIMM, the turbulence profile for the tomography in WFAO systems should be estimated from its WFSs, not from a different system at a different site, such as the MASS and DIMM. Otherwise, we would mis-estimate the ground layer, which has a large contribution of more than 50 per cent of the total $C_{N}^{2}$ at Maunakea, and it has a large impact on the performance of WFAO systems.

\subsection{Is the outer scale biased?}

The FWHMs of PSFs without AO correction, referred to as seeinglimited FWHMs, are affected by the outer scale because the amount of tip/tilt components depends on the outer scale. In other words, the outer scale can be evaluated from the observed seeing-limited FWHMs by comparing it with the seeing assuming the Kolmogorov power spectra.

In Tokovinin (2002), the approximation of the ratio of the seeinglimited FWHMs assuming the von Karman power spectrum to that assuming the Kolmogorov power spectra is investigated though a numerical simulation and it is given as

$$
\left(\frac{\epsilon_{\mathrm{vK}}}{\epsilon_{\mathrm{Kol}}}\right)^{2} \approx 1-2.183\left(\frac{r_{0}}{\mathcal{L}_{0}}\right)^{0.356} .
$$

Here, $\epsilon_{\mathrm{vK}}$ and $\epsilon_{\mathrm{Kol}}$ are seeing-limited FWHMs at a given wavelength $\lambda$ assuming von Karman and Kolmogorov power spectrum, respectively. Using equation (13) and $\epsilon_{\mathrm{Kol}}=0.98 \lambda / r_{0}$, we can obtain a relation between $\epsilon_{\mathrm{vK}, \lambda}$ and $r_{0, \lambda}$ at a given wavelength $\lambda$ as

$\epsilon_{\mathrm{vK}, \lambda}=0.98 \frac{\lambda}{r_{0, \lambda}} \sqrt{1-2.183\left(\frac{r_{0, \lambda}}{\mathcal{L}_{0}}\right)^{0.356}}$,

where $r_{0, \lambda}$ can be computed from $r_{0}$ at $500 \mathrm{~nm}$ measured by the SLODAR using a relation $r_{0} \propto \lambda^{1.2}$.

We compare the seeing-limited FWHMs of $H$-band PSFs observed by RAVEN with those predicted from $r_{0, \lambda}$ estimated by the SLODAR using equation (14) to evaluate $\mathcal{L}_{0}$ in Fig. 9. The grey points in the figure show the seeing-limited FWHMs measured from on-sky PSFs at the $H$ band by a fit of an elliptical Moffat 


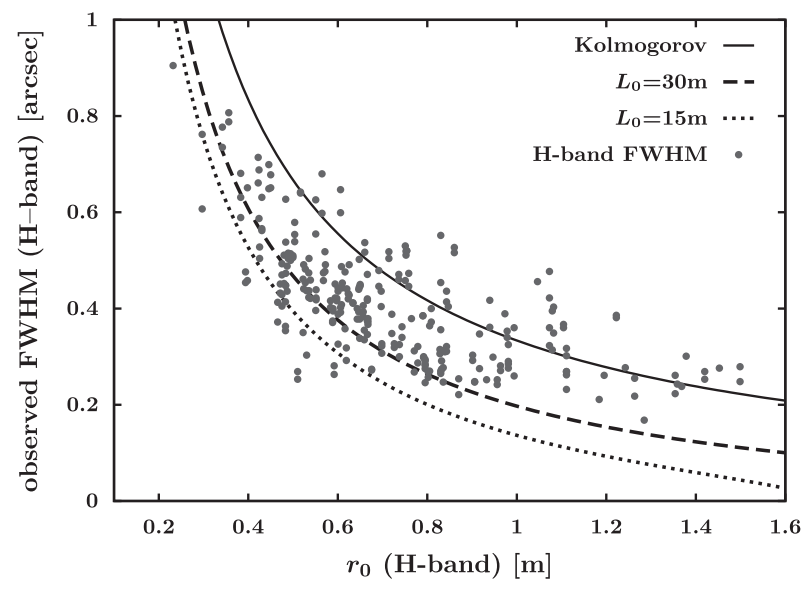

Figure 9. Seeing-limited FWHMs of $H$-band PSFs as a function of $r_{0}$ at the $H$-band. The seeing-limited FWHMs observed by RAVEN are represented by grey points. The black lines are the relation predicted from equation (14) with different $\mathcal{L}_{0}: \mathcal{L}_{0}=\infty$ (Kolmogorov; solid line), $\mathcal{L}_{0}=30 \mathrm{~m}$ (dashed line) and $\mathcal{L}_{0}=15 \mathrm{~m}$ (dotted line).

function. We exclude the elongated PSFs with the FWHM ratio of minor axis to major axis smaller than 0.6 , which is 2 per cent of all the seeing-limited FWHMs, to reduce the bias due to the tip/tilt induced by the telescope guiding error, wind shake and vibration. Furthermore, the FWHMs on the minor axis are used for Fig. 9 to minimize this effect. The average value of the FWHM ratio is 0.83 , and the bias due to the tip/tilt error should be small. The back lines show the prediction from equation (14) with different outer scales. According to Fig. 9, most of the on-sky seeing-limited FWHMs have good agreement with the prediction with $\mathcal{L}_{0}>30 \mathrm{~m}$. This value is larger than the median value of $25.5 \mathrm{~m}$ estimated by the SLODAR, and it indicates that the estimates of the outer scale from the SLODAR are possibly biased to two to three times the telescope aperture.

The outer scale has a large impact on the turbulence strength, especially on the strength of the low-order modes, and so the outer scale strongly affects the design of AO systems, such as the dynamic range of deformable mirrors (DMs) and WFSs and the predictions of AO performance based on numerical simulations. Therefore, further measurements of $\mathcal{L}_{0}$ from different methods are required to estimate the actual outer scale for designing future $\mathrm{AO}$ systems at Maunakea.

The other factor that should be discussed is the impact from the bias effect of the outer scale in terms of tomography for WFAO systems. As mentioned previously, the outer scale mainly affects the tip/tilt modes of the phase aberration. The tomography method, which controls the low- and high-order aberration separately, as proposed in Gilles \& Ellerbroek (2008), can help to reduce the effect from the biased outer scale. At high altitudes, because of the larger meta-pupil size in the atmospheric turbulence volume, the outer scale can affect the higher-order modes of the phase distortion than the tip/tilt modes and cause different tilt anisoplanatism over the field. However, if the SLODAR has the ability to sense larger outer scales at high altitudes because of the larger meta-pupil, the outer-scale effect at high altitudes can be taken into account in tomography.

In the case of ELTs with a primary aperture larger than $30 \mathrm{~m}$, we will be able to sense the outer scale roughly up to $100 \mathrm{~m}$ with the SLODAR method, which is much larger than the typical outer scale (20-30 m) observed at some sites. Therefore, the impact from

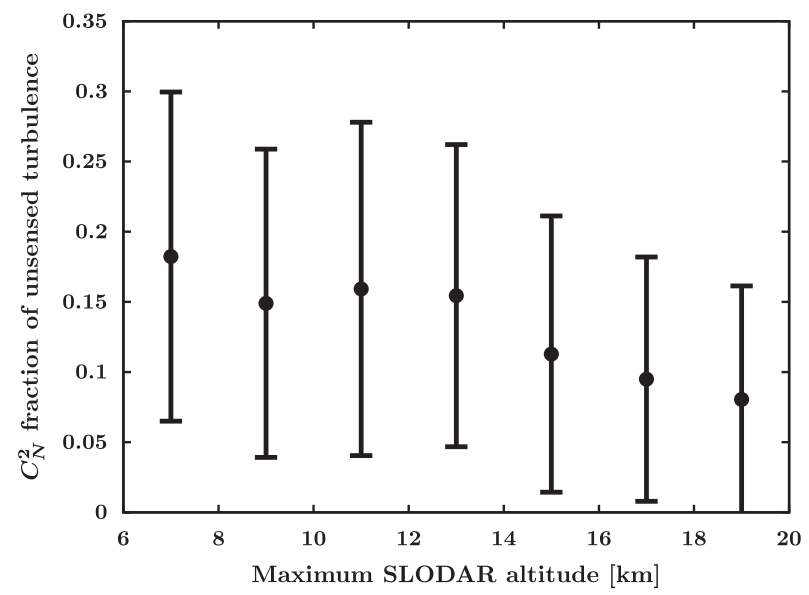

Figure 10. Median $C_{N}^{2}$ fraction of the unsensed turbulence as a function of the maximum altitude $h_{\max }$. The median fraction is computed in each $2-\mathrm{km}$ altitude bin from 6 to $20 \mathrm{~km}$, and the error bars represent standard deviations.

the bias effect in the SLODAR is much smaller compared to cases with current 8 -m class telescopes.

\subsection{Unsensed turbulence}

Currently, the unsensed turbulence is not taken into account in the tomography because the altitude of the unsensed turbulence cannot be estimated by the SLODAR method. It would be possible to include the unsensed turbulence in the tomography by assuming a typical altitude for the unsensed turbulence. However, because the projected pupils in GS directions do not overlap at the unsensed altitude, the unsensed turbulence is hard to reconstruct even if we know its altitude and there might be only small improvement in the tomographic performance by including the unsensed turbulence. This should be investigated by numerical simulation and tests with onsky data. Also, in order to know the typical altitude of the unsensed turbulence, turbulence characterizations that can measure the turbulence higher than altitudes measured by our SLODAR are required. Information regarding the unsensed turbulence could also be useful for post-processing, such as diagnosing the AO performance and PSF reconstruction.

Fig. 10 shows the median $C_{N}^{2}$ fraction of the unsensed turbulence as a function of the maximum altitude $h_{\max }$ that can be sensed by the cross-correlations. Although the fraction of the unsensed turbulence depends strongly on the vertical profile of $C_{N}^{2}$, the median value decreases with $h_{\max }$. As $h_{\max }=6-8 \mathrm{~km}$, roughly 20 per cent of the turbulence is unsensed by the SLODAR. Even as turbulence up to $18-20 \mathrm{~km}$ is detected by the SLODAR, 8 per cent of the turbulence is unsensed. This unsensed turbulence directly affects the performance of tomography and MOAO correction of RAVEN. Therefore, the unsensed turbulence should be considered in the evaluation of the on-sky MOAO performance of RAVEN. In ELTs, the effect of the unsensed turbulence becomes smaller because $h_{\max }$ of the SLODAR increases with the telescope aperture diameter.

It should be noted that the unsensed turbulence estimation cannot be achieved by our SLODAR without NGS. In the case with LGSs, the $C_{N}^{2}$ unsensed turbulence detected by the autocorrelation depends on altitude because of the cone effect of LGSs. Some current and future WFAO systems only have LGSs (and tip/tilt NGSs) and so more work is needed to evaluate the unsensed turbulence with LGSs. 


\section{CONCLUSION}

In this paper, we present the fitted-SLODAR method to estimate the vertical profiles of $C_{N}^{2}$ and the outer scale of the atmospheric turbulence using autocorrelations and cross-correlations of slopes from multiple WFSs in WFAO systems. The analytical partial derivatives of slope correlations with respect to $r_{0}$ and $\mathcal{L}_{0}$ are developed and plugged in the form of the Jacobian to solve for the non-linear model-fit criterion minimization for the SLODAR. Also, this SLODAR method can evaluate the unsensed turbulence, which cannot be sensed by the triangulation of the SLODAR. Finally, $C_{N}^{2}$ and $\mathcal{L}_{0}$ profiles at Maunakea are estimated by the fitted-SLODAR from on-sky telemetry data taken with multiple SH-WFSs in RAVEN during 12 nights of the RAVEN on-sky observations.

The main findings in this paper are as follows.

(i) From a total of 12 nights in the summer season, a large ground $C_{N}^{2}$ fraction of 54.3 per cent is found with the median estimated seeing of 0.460 arcsec. This median seeing value is below other results for Maunakea (0.602 arcsec in Els et al. 2009; 0.684 arcsec in Miyashita et al. 2004).

(ii) The mean profile of $C_{N}^{2}$ indicates that there is strong turbulence at the ground and weak turbulence at $8 \mathrm{~km}$. This profile is in good agreement with the mean $C_{N}^{2}$ profile estimated by the CFHT MASS-DIMM during the RAVEN observations, except for the ground layer. The $C_{N}^{2}$ difference in the ground layer suggests that the ground layer depends strongly on the location. Dome seeing can also affect this difference in the ground layer. The relatively weaker ground layer we have observed contributes to the good seeing conditions during our observation runs.

(iii) The $C_{N}^{2}$ values at each altitude bin estimated by the SLODAR marginally correlate with those from the MASS-DIMM. However, the correlations have large dispersion because of contamination from other altitude bins, especially the $2-\mathrm{km}$ bin. The correlation of $C_{N}^{2}$ for the ground layer has a dispersion larger than that for high altitudes $(h>1.5 \mathrm{~km})$, which suggests that high-altitude turbulence is relatively common for a large field of sky whereas the ground layer depends on the location of the telescope.

(iv) The median $C_{N}^{2}$ fraction of the unsensed turbulence is 11.5 per cent. This fraction decreases with the maximum altitude $h_{\max }$ that can be sensed by the cross-correlation: 18 per cent at $h_{\max }=$ 6-8 $\mathrm{km}$ and 7 per cent at $h_{\max }=18-20 \mathrm{~km}$.

(v) The median value of the outer scale is $25.5 \mathrm{~m}$ and the outer scale increases with altitude, which is consistent with the other results. However, the FWHM of on-sky PSFs in the $H$ band taken by RAVEN suggests that outer scales are larger than $30 \mathrm{~m}$, which means that the estimates of $\mathcal{L}_{0}$ from the SLODAR might be biased towards two to three times the telescope aperture because of the blindness of the SLODAR to large outer scales.

These new processed profiles are very useful for understanding and improving the performance of RAVEN. In particular, the outer-scale profile might have a large impact on the tip/tilt angular anisoplanatism over the field and therefore on tomography. Also, the outer scale affects the estimation of $C_{N}^{2}$ : to represent an optical path difference, the $C_{N}^{2}$ should be larger as $\mathcal{L}_{0}$ decreases. These effects will be more critical for tomographic systems in ELTs where the size of the primary mirror is comparable to the typical outer-scale size.

A possible improvement in our SLODAR is to profile the dome seeing. The dome seeing is suggested to have a very small $\mathcal{L}_{0}$ (Guesalaga et al. 2016), and usually the dome seeing is considered to largely contribute to the ground layer. The dome seeing can be estimated by assuming two turbulent modes with different outer scale at the ground, and this can easily be taken into account in our theoretical model.

Another point to be improved is the estimation of wind speed and direction. Recently, some algorithms for predictive atmospheric turbulence tomography in WFAO systems were proposed (Correia et al. 2014; Ono et al. 2016), which require monitoring the wind speed and direction at each altitude. The estimation of the wind information of the turbulence can be achieved using temporal correlation of measurements from multiple WFSs (Ono et al. 2016). Our theoretical model for slope correlations can include the wind speed and direction, and the wind speed and direction can be automatically estimated by fitting this theoretical model to the observed temporal correlation with different time delays.

\section{ACKNOWLEDGEMENTS}

This work is supported by the Japan Society for the Promotion of Science (JSPS) Grant-in-Aid for JSPS Fellows (15J02510) and the A*MIDEX project (no. ANR-11-IDEX-0001-02) funded by the 'Investissements d'Avenir' French Government programme, managed by the French National Research Agency (ANR). MA is supported by JSPS Grant-in-Aid for Scientific Research (B) (26287027). The authors thank Olivier Martin for many discussions, the referee for his/her comments and suggestions and the staff members of the Subaru telescope for their support.

\section{REFERENCES}

Andersen D. R., Bradley C., Gamroth D., Kerley D., Lardière O., Véran J.-P., 2014, Proc. SPIE, 9148, 91485K

Butterley T., Wilson R. W., Sarazin M., 2006, MNRAS, 369, 835

Correia C., Jackson K., Véran J.-P., Andersen D., Lardière O., Bradley C., 2014, J. Opt. Soc. Am. A, 31, 101

Correia C. M., Jackson K., Véran J.-P., Andersen D., Lardière O., Bradley C., 2015, Appl. Opt., 54, 5281

Cortés A., Neichel B., Guesalaga A., Osborn J., Rigaut F., Guzman D., 2012, MNRAS, 427, 2089

Davidge T. J., Andersen D. R., Lardière O., Bradley C., Blain C., Oya S., Akiyama M., Ono Y. H., 2015, ApJ, 811, 133

Davidge T. J. et al., 2016, AJ, 152, 173

Els S. G., Travouillon T., Schöck M., Riddle R., Skidmore W., Seguel J., Bustos E., Walker D., 2009, PASP, 121, 527

Gilles L., Ellerbroek B. L., 2008, J. Opt. Soc. Am. A, 25, 2427

Guesalaga A., Neichel B., Cortés A., échet C., Guzmán D., 2014, MNRAS, 440, 1925

Guesalaga A., Neichel B., Correia C., Butterley T., Osborn J., Masciadri E., Fusco T., Sauvage J.-F., 2016, Proc. SPIE, 9909, 99093C

Herriot G. et al., 2014, Proc. SPIE, 9148, 914810

Lamb M. et al., 2016, MNRAS, in press (doi:10.1093/mnras/stw2865)

Lardière O. et al., 2014, Proc. SPIE, 9148, 91481G

Maire J., Ziad A., Borgnino J., Martin F., 2007, MNRAS, 377, 1236

Martin O., Gendron É., Rousset G., Vidal F., 2012, Proc. SPIE, 8447, 84472A

Martin O. A. et al., 2016, Proc. SPIE, 9909, 99093P

Miyashita A., Takato N., Usuda T., Uraguchi F., Ogasawara R., 2004, Proc. SPIE, 5489, 207

Neichel B. et al., 2014, MNRAS, 440, 1002

Ono Y. H., Akiyama M., Oya S., Lardiére O., Andersen D. R., Correia C., Jackson K., Bradley C., 2016, J. Opt. Soc. Am. A, 33, 726

Roddier F., 1981, The Effects of Atmospheric Turbulence in Optical Astronomy. North-Holland, Amsterdam

Ströbele S. et al., 2012, Proc. SPIE, 8447, 844737

Thatte N. A. et al., 2014, Proc. SPIE, 9147, 914725

Tokovinin A., 2002, PASP, 114, 1156

Tokovinin A., Vernin J., Ziad A., Chun M., 2005, PASP, 117, 395 
Valenzuela J. et al., 2014, Proc. SPIE, 9148, 91481X

Vidal F. et al., 2014, A\&A, 569, A16

Wang L., Schöck M., Chanan G., 2008, Appl. Opt., 47, 1880

Wilson R. W., 2002, MNRAS, 337, 103

Ziad A., Schöck M., Chanan G. A., Troy M., Dekany R., Lane B. F., Borgnino J., Martin F., 2004, Appl. Opt., 43, 2316

\section{APPENDIX: PARTIAL DEVIATION OF THE} VON KARMAN STRUCTURE FUNCTION

Here, we show how to compute the partial deviation of the von Karman structure function in equation (6). With respect to $r_{0}$, it is easily computed because $r_{0}$ is included in the first set of parentheses as

$\frac{\partial D_{\phi}(\rho)}{\partial r_{0}}=-\frac{5}{3} r_{0}^{-1} D_{\phi}(\rho)$.
The partial deviation with respect to $\mathcal{L}_{0}$ is more complex than the case of $r_{0}$. To deal with deviation of the modified Bessel function of the second kind, we use the following expression,

$\frac{\partial}{\partial x}\left[x^{v} K_{v}(x)\right]=-x^{v} K_{v-1}(x)$

where $x$ is $2 \pi \rho / L_{0}$ in our case. The final formulation is given as

$$
\begin{aligned}
\frac{\partial D_{\phi}(\rho)}{\partial L_{0}}= & \frac{5}{3} L_{0}^{-1} D_{\phi}-0.17253 \frac{2^{1 / 6}}{\Gamma(5 / 6)}\left(\frac{L_{0}}{r_{0}}\right)^{5 / 3} \\
& \times\left(\frac{2 \pi \rho}{L_{0}}\right)^{11 / 6} L_{0}^{-1} K_{-1 / 6}\left(\frac{2 \pi \rho}{L_{0}}\right) .
\end{aligned}
$$

This paper has been typeset from a $\mathrm{T}_{\mathrm{E}} \mathrm{X} / \mathrm{L} \mathrm{T} \mathrm{E} \mathrm{X}$ file prepared by the author. 